\title{
Impacto de las TIC: desafíos y oportunidades de la Educación Superior frente al COVID-19
}

Fecha de recepción : 10-01-2021 • Fecha de aceptación: 05-04-2021 • Fecha de publicación: 10-05-2021

\author{
Janeth Pilar Díaz Vera ${ }^{1}$ \\ Universidad de Guayaquil, Ecuador \\ janeth.diazv@ug.edu.ec \\ https://orcid.org/0000-0001-8750-0216
}

Alicia Karina Ruiz Ramírez ${ }^{2}$

Universidad de Guayaquil, Ecuador

alicia.ruizram@ug.edu.ec

https://orcid.org/0000-0002-3038-045X

Carolina Egüez Cevallos ${ }^{3}$

Universidad de Guayaquil, Ecuador

carolina.eguezc@ug.edu.ec

https://orcid.org/0000-0003-0226-3026

\section{Resumen}

El objetivo de este estudio fue analizar el impacto en el uso de las TIC para el desarrollo de sus actividades académicas en la modalidad de clases en línea de la Universidad de Guayaquil, en tiempo de pandemia por el COVID-19, crisis de salud global que incluye al Ecuador. Esta investigación fue de campo y su diseño no experimental transversal. La información se recolectó a través de un cuestionario de preguntas de diferentes tipos, la confiabilidad de este instrumento se hizo a través del coeficiente Alfa de Cronbach, los resultados de las encuestas se procesaron con el programa Excel y se presentaron en tablas de frecuencia. La población utilizada fue de 2138 docentes y 57567 estudiantes de las 17 facultades de la Universidad de Guayaquil. La muestra fue de tipo no probabilística o dirigida, con un total de 246 docentes y 276 de estudiantes. Los resultados indican que, para los docentes que participaron en el estudio, el uso de Tecnologías de Información y Comunicación (TIC) 
son de suma importancia para el desarrollo de la docencia, especialmente en las clases virtuales. Sin embargo, consideran que deben mejorarse los procesos de capacitación en el uso de estas tecnologías. Se concluye que tanto docentes y estudiantes reconocen que no pueden separar las tecnologías del proceso enseñanza - aprendizaje, pero es clave una adecuada capacitación de las competencias digitales para su uso y máximo aprovechamiento.

Palabras clave: tecnologías educativas, educación superior, competencias digitales, procesos académicos, COVID-19.

\begin{abstract}
The objective of this study was to analyze the impact on the use of ICT for the development of their academic activities in the modality of online classes at the University of Guayaquil, in times of the COVID-19 pandemic, a global health crisis that includes Ecuador. This research was in the field and its design was not cross-sectional. The information was collected through a questionnaire of different types of questions, the reliability of this instrument was made through Cronbach's Alpha coefficient, the results of the surveys were processed with the Excel program and presented in frequency tables. The population used was 2,138 teachers and 57,567 students from the 17 faculties of the University of Guayaquil. The sample was non-probabilistic or directed, with a total of 246 teachers and 276 students. The results indicate that, for the teachers who participated in the study, the use of Information and Communication Technologies (ICT) is of utmost importance for the development of teaching, especially in virtual classes. However, they consider that training processes in the use of these technologies should be improved. It is concluded that both teachers and students recognize that they cannot separate technologies from the teaching - learning process, but adequate training of digital skills is essential for their use and maximum use.
\end{abstract}

Keywords: educational technologies, higher education, digital skills, academic processes, COVID-19. 


\section{Introducción}

A finales del año 2019, por causa de un repentino brote de un enemigo imperceptible a la vista nombrado COVID-19, el cual tuvo origen en la ciudad de Wuhan, China, y se ha esparcido en todos los países del mundo en un corto lapso de tiempo; obligó a cerrar escuelas y universidades, perjudicando a todos los estudiantes a nivel mundial e impulsado un despliegue acelerado de soluciones de educación a distancia, para asegurar la continuidad pedagógica.

Esta crisis mundial ha desencadenado un replanteamiento de la prestación de servicios educativos a todos los niveles, el uso intensivo de todo tipo de plataformas y recursos tecnológicos para garantizar la continuidad del aprendizaje es el experimento más audaz en materia de tecnología educativa, aunque inesperado y no planificado. Las actividades en el régimen educativo privado y público se han visto afectadas por la pandemia de la COVID-19, ocasionando la suspensión de todas las unidades educativas y aplazando el inicio del siguiente período lectivo, dado que no se encontraban listos para el distanciamiento social, que conllevó a implementar medidas de confinamiento. Por estos motivos, las unidades educativas recurrieron a la experimentación de nuevas estrategias enfocadas a la educación a distancia para seguir con el proceso de enseñanzaaprendizaje (López, y otros, 2020).

La educación superior no es una excepción, aunque a este nivel la tecnología digital ha tenido el mayor impacto en las últimas décadas. En Ecuador, la universidad más grande que existe es la Universidad de Guayaquil (UG), esta cuenta con más de 57.000 estudiantes registrados; su medida de cambio fue la transición de la modalidad presencial a virtual, implementando las Tecnologías de la Información y Comunicación (TIC), entre estas se puede hacer mención a herramientas como Zoom, una aplicación de videoconferencia y audio conferencia para que los profesores y estudiantes puedan realizar las clases virtuales de manera interactiva; y Moodle, que es una aplicación de gestión y creación de espacios para el aprendizaje.

En esta transición, la Universidad de Guayaquil, al igual que las demás Instituciones de Educación Superior (IES), ha tenido múltiples obstáculos, desde la baja conectividad y la falta de contenido en línea, alineado con los planes de estudio nacionales, hasta un profesorado no preparado para esta "nueva normalidad". La Organización de Naciones Unidas para la Educación, Ciencia y Cultura (UNESCO, 2020) plantea que "casi 1100 millones de estudiantes y jóvenes de todo el mundo están afectados por el cierre de escuelas y universidades debido al brote de la COVID-19".

El Vicerrectorado de Formación Académica y Profesional (VIFAP) de la Universidad de Guayaquil, decidió redactar un memorándum y enviarlo a todos los decanos de todas las facultades que conforman la institución en el cual se adjunta la "Guía Metodológica Académica Modalidad en Línea - UG 2020", en este se disponen las directrices, lineamientos y conceptos fundamentales, dirigido a todos los docentes de la institución, y la implementación del proceso de enseñanzaaprendizaje en modalidad en línea, haciendo uso adecuado de las herramientas tecnológicas que dispone la institución (Universidad de Guayaquil, 2020a, p. 3).

El objetivo principal del estudio es analizar el impacto del uso de las TIC en la modalidad de clases en línea de la Universidad de Guayaquil durante el tiempo de la COVID-19, a través de 
un estudio de campo, bibliográfico y exploratorio. El enfoque de esta investigación es mixto; es decir cualitativo-cuantitativo, para la metodología se han empleado los métodos analítico, inductivo y deductivo. Entre las técnicas para la recolección de la información se ha aplicado la encuesta, y como instrumento se elaboró un cuestionario de 10 preguntas de carácter cerrado, las mismas que fueron creadas usando el formulario de Google, estas encuestas se realizaron a una muestra seleccionada de docentes y estudiantes de las diferentes facultades de la Universidad de Guayaquil.

\subsection{Antecedentes}

La Universidad de Guayaquil, pese a las exigencias académicas requeridas durante varios años, no había realizado un cambio sumamente importante respecto a la aplicación de las TIC, en el 2020, a causa de la pandemia global y al decreto de estado de excepción impuesto por el Gobierno, se hizo necesario implementar cambios para acelerar la transición a la nueva modalidad de educación.

La universidad ha realizado cursos y capacitaciones a los docentes de las diferentes facultades sobre metodologías, diseño curricular y de investigación; pero tanto a los docentes, como estudiantes, no se le han impartido cursos o capacitaciones con respecto al desarrollo de competencias digitales, para que puedan adquirir las habilidades y destrezas en el uso de recursos tecnológicos.

La Constitución de la República de Ecuador (2008), en el Art. 347 numeral 8, establece que será compromiso del Estado "incorporar las tecnologías de la información y comunicación en el proceso educativo y propiciar el enlace de la enseñanza con las actividades productivas y sociales". Así también la Ley Orgánica de Educación Intercultural (2017), en su Art. 6, respalda el uso de las TIC en los procesos educativos para el desarrollo de actividades productivas o sociales. Por otro lado, la Ley Orgánica de Educación Intercultural (2011), hace énfasis en que "el desarrollo profesional es un proceso permanente e integral de actualización psicopedagógica y en ciencias de la educación" (Art. 112).

Las IES, de acuerdo con la Ley Orgánica de Educación Superior del Ecuador y su Reglamento, hacen énfasis en que la aplicación de TIC debe estar acorde con el desarrollo científico tecnológico de la sociedad del siglo XXI. La Universidad de Guayaquil, durante los últimos años se ha visto fortalecida con la implementación de banda ancha para la accesibilidad al Internet de toda la comunidad universitaria, por ello, a través de su página web se puede obtener información relacionada con la gestión de las distintas funciones sustantivas.

El problema surge debido a que la institución caso de estudio no había realizado por muchos años un cambio tan importante en la tecnología, a pesar de que las exigencias académicas lo requerían, en el 2020 debido a la pandemia global de la COVID-19 y al decreto de estado de excepción que impuso el gobierno, ven necesario implementar cambios para acelerar la transición a la nueva modalidad de educación, donde las TIC se han convertido en variables fundamentales en la educación, permitiendo la creación de entornos virtuales, ampliando el acceso a la información y eliminando las barreras espacios-temporales entre los estudiantes y docentes. 


\section{2 Generalidades sobre el uso de las TIC en Instituciones de Educación Superior}

Las TIC son aquellas que permiten crear nuevas maneras de comunicar, haciendo uso de la microelectrónica, informática y telecomunicaciones mediante herramientas tecnológicas y de comunicación, con el objetivo de conceder acceso, emisión y procesamiento de la información. Estas son herramientas muy valiosas en esta época de la información y del conocimiento, por este motivo la comunidad se ve en la obligación de incorporarlas en el régimen educativo, para que así las futuras generaciones puedan estar familiarizadas con el uso de las TIC, con el fin de facilitar a la sociedad herramientas que aceleren el procesamiento de la información (Contreras, Pabón, \& Ríos, 2017)

En un proceso de enseñanza-aprendizaje juegan un papel importante las tecnologías de la información y comunicación como apoyo en la interacción con actividades didácticas que integran lo visual, novedoso e interactivo; incentiva el uso de aplicaciones, plataformas y redes sociales; promueve nuevas formas de enseñanza; facilita la búsqueda de información y comunicación, el desarrollo de actividades prácticas del quehacer docente como las videoconferencias, las cuales constituyen un servicio que permite poner en contacto a un grupo de personas mediante sesiones interactivas para que puedan ver y escuchar una conferencia (García et al., 2018, párr. 17).

Las TIC, dentro del ámbito educativo, son un complemento para el desarrollo del proceso de enseñanza-aprendizaje debido a que facilitan la interacción de las actividades didácticas, estimulan el uso plataformas y aplicaciones educativas y permiten el trabajo colaborativo entre diferentes grupos de personas. En la formación educativa superior se han implementado como complemento para el aprendizaje, lo que ha traído varias ventajas como:

- Aprovechar los recursos tecnológicos

- Autoaprendizaje

- Interacción continua con programas u otras personas conectadas

- Herramientas y material de estudio gratuito

De la misma manera, también ha traído desventajas como:

- Información no confiable

- Distracciones en Internet

- Cansancio visual y otros problemas físicos

- Aprendizajes incompletos

- Pérdida de tiempo 
El objetivo de un plan de aprendizaje, basado en la apropiación de las TIC, debe iniciar a partir del desarrollo de múltiples competencias en el ámbito educativo. Pero en este caso se puede partir de las competencias vinculadas con el diseño, implementación y evaluación de los escenarios educativos, donde interviene el uso de las TIC, como aparece en la Tabla 1.

Tabla 1.

Competencias de las TIC desde la dimensión pedagógica

\begin{tabular}{|l|l|}
\hline Competencia & Descripción \\
\hline Diseño & $\begin{array}{l}\text { Es la capacidad de planificar y organizar los elementos que posibilitan } \\
\text { la creación de entornos educativos, basados en las TIC, para apoyar el } \\
\text { aprendizaje del estudiante. }\end{array}$ \\
\hline Implementación & $\begin{array}{l}\text { Es la capacidad de poner en marcha el diseño y planificación de los } \\
\text { entornos educativos. Esto se refleja en las prácticas que utilizan los } \\
\text { docentes para la enseñanza. }\end{array}$ \\
\hline Evaluación & $\begin{array}{l}\text { Es la capacidad que permite al docente evaluar el nivel de aprendizaje } \\
\text { que se ha obtenido en los estudiantes al implementar las TIC en sus } \\
\text { actividades educativas. }\end{array}$ \\
\hline
\end{tabular}

Fuente: elaboración propia

\subsection{Niveles de apropiación de las TIC}

- Nivel de Integración. En este nivel se tiene la idea de las TIC como instrumentos que ayudan a la comunicación, difusión y exposición de contenido. Se pueden encontrar prácticas de digitalización, de contenidos educativos, así como el traspaso de documentos en papel a digital.

Ejemplo: al realizar evaluaciones en un ambiente digital, se pueden realizar diferentes tipos de preguntas (opción múltiple, verdadero o falso, etc.), lo cual permite que la calificación sea instantánea, así los estudiantes pueden verificar las respuestas correctas o erróneas de forma rápida.

- Nivel de reorientación. Este nivel se caracteriza particularmente porque los docentes emplean herramientas tecnológicas en actividades educativas específicas para motivar a la participación de los estudiantes. Las TIC dejan de ser vistas como una herramienta que proporciona grandes cantidades de información y pasan a ser adoptadas como una herramienta en la construcción del conocimiento.

Ejemplo: creación participativa de un blog para complementar el contenido visto en las clases. Los maestros solicitan a sus estudiantes que realicen un blog o sitio web con la información previamente obtenida de temas tratados en clases, esto permite intercambiar puntos de vista y contenidos digitales entre el alumnado.

- Nivel de Evolución. Las TIC ayudan a expandir las capacidades humanas para representar, procesar y transmitir información. El docente puede medir el conocimiento adquirido por los estudiantes, así mismo evaluar el nivel de intercambio de información entre docente/estudiante, estudiante/estudiante, docente/docente y grupos de investigación. 
Ejemplo: el maestro utiliza de manera creativa herramientas virtuales que permitan la interacción del estudiante con el objeto de estudio con el fin de que desarrolle pensamiento o razonamiento crítico del contenido estudiado.

\subsection{Competencias digitales}

Se pueden definir como un conjunto de habilidades, estrategias o conocimientos que se necesitan para hacer un correcto uso de las TIC. Estas competencias facilitan la comunicación, obtención de información, seguridad digital y la creación e intercambio de contenido digital. Las competencias digitales "son asumidas a manera de instrumentos de gran utilidad que permite la movilización de actitudes, conocimientos y procesos; por medio de los cuales los docentes adquieren habilidades para facilitar la transferencia de conocimientos y generar innovación" (Marza y Cruz, 2018, como se citó en Levano et al., 2019).

Estas han adquirido una gran relevancia a nivel educativo debido a que permiten acomodarse a las necesidades actuales, y posibilitan desarrollar una conducta crítica y realista entorno a las tecnologías. Debido al gran uso de las TIC y su cambio constante, los docentes se ven en la necesidad de adquirir competencias digitales, pero estas deben ser estudiadas, profundizadas y actualizadas de manera continua para poder aplicarlo en el proceso de enseñanza.

De acuerdo con Mosquera (2018), "se trata de una competencia dinámica cuyo marco debe ser renovado de manera periódica para adaptarse a los nuevos contextos educativos que las propias tecnologías van generando". Por lo que se debe tomar vital importancia a los cambios que producen las TIC con respecto al marco educativo, para de esta manera estar a la altura de las demandas que estas conllevan.

El objetivo de un plan de aprendizaje, basado en la apropiación de las TIC, debe iniciar a partir del desarrollo de múltiples competencias en el ámbito educativo. Pero en este caso se puede partir de las competencias vinculadas con el diseño, implementación y evaluación de los escenarios educativos donde interviene el uso de las TIC.

\subsection{Clases en línea}

Las clases en línea comprenden el proceso enseñanza-aprendizaje entre estudiantes y docentes en un ambiente totalmente digital con la ayuda de recursos tecnológicos. Se logra tener un ámbito interactivo, es decir, se puede acceder a cualquier hora o lugar. Un ambiente digital es el entorno que permite la interacción a distancia entre usuarios que se conectan a través de una red de computadoras; esta requiere un nivel de independencia y se espera que los estudiantes absorban el contenido, sigan instrucciones, perseveren en la actividad asignada y respondan preguntas; la parte personalizada es que los chicos puedan avanzar a su propio ritmo.

La Tabla 2 muestra la comparación de las clases presenciales versus las clases virtuales. 
Tabla 2.

Comparación de clases presenciales vs clases virtuales

\begin{tabular}{|l|l|}
\hline Clases presenciales & Clases virtuales \\
\hline Requieren de un espacio físico & No requieren espacio físico \\
\hline $\begin{array}{l}\text { Necesidad de desplazamiento a centro de estudio } \\
\text { para asistir a las clases }\end{array}$ & $\begin{array}{l}\text { Se puede acceder a las clases o cursos } \\
\text { virtuales desde cualquier lugar donde el usuario } \\
\text { se encuentre }\end{array}$ \\
\hline Horarios estrictos o rígidos & Flexibilidad en los horarios \\
\hline $\begin{array}{l}\text { Existe interacción física por parte de las personas } \\
\text { que conforman un aula }\end{array}$ & $\begin{array}{l}\text { No hay interacción física por parte de las } \\
\text { personas que conforman una clase virtual }\end{array}$ \\
\hline Contenido educativo estático & $\begin{array}{l}\text { Contenidos educativos actualizados y dinámicos } \\
\text { a través de medios electrónicos }\end{array}$ \\
\hline Enseñanza tradicional por parte de un docente & $\begin{array}{l}\text { El docente utiliza herramientas tecnológicas lo } \\
\text { cual produce una enseñanza dinámica }\end{array}$ \\
\hline $\begin{array}{l}\text { Limitaciones geográficas para acceder a la } \\
\text { educación }\end{array}$ & $\begin{array}{l}\text { No existen limitaciones geográficas porque } \\
\text { se puede acceder a la educación por parte de } \\
\text { cualquier docente o instructor en cualquier lugar } \\
\text { que se encuentre }\end{array}$ \\
\hline $\begin{array}{l}\text { No requieren de herramientas tecnológicas o } \\
\text { digitales para su implementación }\end{array}$ & $\begin{array}{l}\text { Requieren de herramientas tecnológicas o } \\
\text { digitales para su implementación }\end{array}$ \\
\hline $\begin{array}{l}\text { No es indispensable tener conocimientos básicos } \\
\text { acerca del uso de la tecnología }\end{array}$ & $\begin{array}{l}\text { Es indispensable tener conocimientos básicos } \\
\text { acerca del uso de la tecnología }\end{array}$ \\
\hline
\end{tabular}

Fuente: elaboración propia

Pero, así como traen ventajas, también tiene desventajas:

- Requiere constancia y disciplina

- Demasiado tiempo frente a una pantalla

- Dificultades técnicas

- Falta de recursos tecnológicos

Las clases en línea se dividen en algunos tipos de aprendizajes, en la Tabla $\mathbf{3}$ se tiene esta clasificación: 
Tabla 3.

Tipos de aprendizajes de clases en línea

\begin{tabular}{|l|l|}
\hline Tipo & Definición \\
\hline $\begin{array}{l}\text { Modelo de } \\
\text { rotación }\end{array}$ & $\begin{array}{l}\text { Los estudiantes participan del aprendizaje virtual desde el salón de clases o desde } \\
\text { su hogar. Reciben clases en un aula tradicional, pero utilizan la tecnología como } \\
\text { complemento para el aprendizaje. }\end{array}$ \\
\hline Modelo flexible & $\begin{array}{l}\text { Las actividades en línea son el mayor aporte de conocimiento, pero este modelo } \\
\text { permite adaptarse a los otros modelos. }\end{array}$ \\
\hline $\begin{array}{l}\text { Modelo a la } \\
\text { carta }\end{array}$ & $\begin{array}{l}\text { Son cursos impartidos únicamente de manera virtual. Estas capacitaciones formar } \\
\text { parte de la catedra impartida en clase. }\end{array}$ \\
\hline $\begin{array}{l}\text { Modelo virtual } \\
\text { enriquecido }\end{array}$ & $\begin{array}{l}\text { Son cursos que se realizan la mayor parte a distancia, pero requieren la ayuda de } \\
\text { la modalidad tradicional. }\end{array}$ \\
\hline $\begin{array}{l}\text { Modelo } \\
\text { únicamente en } \\
\text { línea }\end{array}$ & $\begin{array}{l}\text { Como lo indica su nombre es aquel donde todos los cursos se realizan a distancia } \\
\text { o de manera virtual, con la participación de un docente o varios docentes } \\
\text { conectados de la misma manera. }\end{array}$ \\
\hline
\end{tabular}

Fuente: elaboración propia

\section{Metodología}

El estudio se realizó en las 17 facultades y 48 carreras del período académico 2020- 2021, ciclo I de la Universidad de Guayaquil. Para la matrícula del período académico julio a octubre 2020, en la Universidad de Guayaquil se matricularon 57567 estudiantes, tal como se puede observar en la Tabla 5. Su planta docente actualmente está conformada por 2138 docentes que incluye a docentes con nombramiento y contrato de las diferentes facultades de la Universidad, como se detalla en la Tabla 4. 
Tabla 4.

Población de la Investigación - Docentes

\begin{tabular}{|c|c|c|}
\hline No & FACULTAD & CANT. \\
\hline 1 & Arquitectura Y Urbanismo & 79 \\
\hline 2 & Ciencias Administrativas & 335 \\
\hline 3 & Ciencias Agrarias & 27 \\
\hline 4 & Ciencias Económicas & 78 \\
\hline 5 & Ciencias Matemáticas y Físicas & 160 \\
\hline 6 & Ciencias Medicas & 470 \\
\hline 7 & Ciencias Naturales & 47 \\
\hline 8 & Ciencias Psicológicas & 48 \\
\hline 9 & Ciencias Químicas & 41 \\
\hline 10 & Comunicación Social & 114 \\
\hline 11 & Educación Física Deporte y Recreación & 39 \\
\hline 12 & Filosofía, Letras y Ciencias de la Educación & 245 \\
\hline 13 & Ingeniería Industrial & 116 \\
\hline 14 & Ingeniería Química & 92 \\
\hline 15 & Jurisprudencia Ciencias Sociales y Políticas & 118 \\
\hline 16 & Medicina Veterinaria Y Zootecnia & 21 \\
\hline 17 & Odontología & 103 \\
\hline 18 & Arquitectura Y Urbanismo I Ciencias Económicas & 1 \\
\hline 19 & Arquitectura Y Urbanismo I Odontología & 1 \\
\hline 20 & Ciencias Agrarias I Comunicación Social & 1 \\
\hline 21 & Ciencias Médicas I Comunicación Social & 1 \\
\hline \multirow[t]{2}{*}{22} & Ciencias Químicas I Ingeniería Industrial & 1 \\
\hline & TOTAL & 2138 \\
\hline
\end{tabular}

Fuente: Sistema Integrado de la Universidad de Guayaquil (SIUG)

Tabla 5.

Población de la Investigación - Estudiantes

\begin{tabular}{|l|l|l|}
\hline No. & FACULTAD & CANT. \\
\hline 1 & Arquitectura y Urbanismo & 1720 \\
\hline 2 & Ciencias Administrativas & 10393 \\
\hline 3 & Ciencias Agrarias & 783 \\
\hline 4 & Ciencias Económicas & 2322 \\
\hline 5 & Ciencias Matemáticas y Físicas & 5437 \\
\hline 6 & Ciencias Medicas & 8302 \\
\hline 7 & Ciencias Naturales & 959 \\
\hline 8 & Ciencias Psicológicas & 2007 \\
\hline 9 & Ciencias Químicas & 1417 \\
\hline 10 & Comunicación Social & 3332 \\
\hline 11 & Educación Física Deporte y Recreación & 1190 \\
\hline
\end{tabular}




\begin{tabular}{|l|l|l|}
\hline 12 & Filosofía, Letras y Ciencias de la Educación & 6658 \\
\hline 13 & Ingeniería Industrial & 4157 \\
\hline 14 & Ingeniería Química & 2765 \\
\hline 15 & Jurisprudencia Ciencias Sociales y Políticas & 3664 \\
\hline 16 & Medicina Veterinaria y Zootecnia & 560 \\
\hline 17 & Odontología & 1897 \\
\hline 18 & Arquitectura y Urbanismo I Ciencias Matemáticas y Físicas & 1 \\
\hline 19 & Ciencias Médicas I Ciencias Psicológicas & 1 \\
\hline 20 & Ciencias Psicológicas I Comunicación Social & 1 \\
\hline & Comunicación Social I Jurisprudencia I Ciencias Sociales y & 1 \\
\hline & Políticas & \\
\hline & TOTAL & 57567 \\
\hline
\end{tabular}

Fuente: Sistema Integrado de la Universidad de Guayaquil (SIUG)

Esta investigación fue de campo, y el diseño no experimental transversal porque se recabó la información en un momento único, y el alcance fue exploratorio -descriptivo (Hernández Sampieri y Mendoza-Torres, 2018). La información se recolectó a través de un cuestionario de preguntas de diferentes tipos, a la cual se le calculó la confiabilidad a través del coeficiente Alfa de Cronbach con un valor de 0,86 , lo que indica que los resultados obtenidos de la aplicación del cuestionario a los docentes y estudiantes son consistentes y coherentes.

Una vez determinada la confiabilidad del cuestionario se procedió a enviar por correo electrónico el link de la encuesta a docentes y estudiantes. Los resultados se procesaron en la herramienta de google form, se generó de manera automática la tabulación en una hoja de Excel y se presentaron en tablas de frecuencia. La población de la investigación estuvo conformada por 246 docentes y 276 estudiantes adscritos a la Universidad de Guayaquil. La muestra fue de tipo no probabilística o dirigida (Hernández Sampieri y Mendoza-Torres, 2018), tomando como criterio aquellos docentes y estudiantes que voluntariamente accedieran a participar en el estudio, lo cual permitió garantizar la franqueza de las respuestas.

Para poder obtener una muestra del total de estudiantes y docentes de la Universidad de Guayaquil se utilizó la siguiente fórmula:

$$
n=\frac{\mathrm{m}}{e^{2}(m-1)+1}
$$

Donde:

m: Tamaño de la población

e: error de estimación

n: Tamaño de la muestra 
Para poder calcular la muestra de la investigación se ha considerado dividirla en dos estratos, que son estudiantes y docentes de la Universidad de Guayaquil.

- Muestra de docentes

$$
\begin{gathered}
\text { Datos } \\
m_{1}: 2138 \\
e: 6 \% \\
n_{1}=\frac{m_{1}}{e^{2}\left(m_{1}-1\right)+1} \\
n_{1}=\frac{2138}{0.06^{2}(2138-1)+1} \\
n_{1}=\frac{2138}{0.0036(2137)+1} \\
n_{1}=\frac{2138}{7.6932+1} \\
n_{1}=\frac{2138}{8.6932} \\
n_{1}=245.93
\end{gathered}
$$

\section{- Muestra de estudiantes}

\section{Datos}

$m 2: 57567$

$e: 6 \%$ 


$$
\begin{gathered}
n_{2}=\frac{m_{2}}{0.06^{2}\left(m_{2}-1\right)+1} \\
n_{2}=\frac{57567}{0.06^{2}(57567-1)+1} \\
n_{2}=\frac{57567}{0.0036(57566)+1} \\
n_{2}=\frac{57567}{207.237+1} \\
n_{2}=\frac{57567}{208.237} \\
n_{2}=276.45
\end{gathered}
$$

\section{Resultados}

La recolección de la información se efectuó a través de muestras tomadas de la población perteneciente a la Universidad de Guayaquil, vía web, mediante el correo electrónico institucional y redes sociales desde 1 al 10 de septiembre del 2020, en las que se incluyen docentes y estudiantes, de todas las facultades y carreras que conforman la institución. La población de la investigación estuvo conformada por 246 docentes y 276 estudiantes adscritos a la institución de Educación Superior.

Los resultados de las encuestas se procesaron en la herramienta de google form y se generó de manera automática la tabulación en una hoja de excel y se presentaron en tablas de frecuencia y gráficos estadísticos para una mejor interpretación y análisis y se aprecian las respuestas de los docentes y estudiantes de la Universidad de Guayaquil. Los resultados evidencian que el 100\% de los encuestados reconocen la relevancia e impacto que tienen las TIC en el ámbito educativo.

\subsection{Análisis e interpretación de resultados de la encuesta de docentes}

Una de las preguntas que se les realizó a los docentes se centró en el nivel que ellos consideraban que tenían en cuanto a la apropiación de las TIC.

En la Tabla 6 y en la Figura 1 podremos ver los niveles indicados, de acuerdo a las opciones establecidas. 
Tabla 6.

Nivel de apropiación de las TIC en docentes

\begin{tabular}{|l|l|l|}
\hline Opción & Docentes encuestados & Porcentaje \\
\hline Muy alto & 35 & $14,2 \%$ \\
\hline Alto & 61 & $24,8 \%$ \\
\hline Intermedio & 119 & $48,4 \%$ \\
\hline Bajo & 27 & $11,0 \%$ \\
\hline Muy Bajo & 4 & $1,6 \%$ \\
\hline Total & 246 & $100 \%$ \\
\hline
\end{tabular}

Fuente: elaboración propia

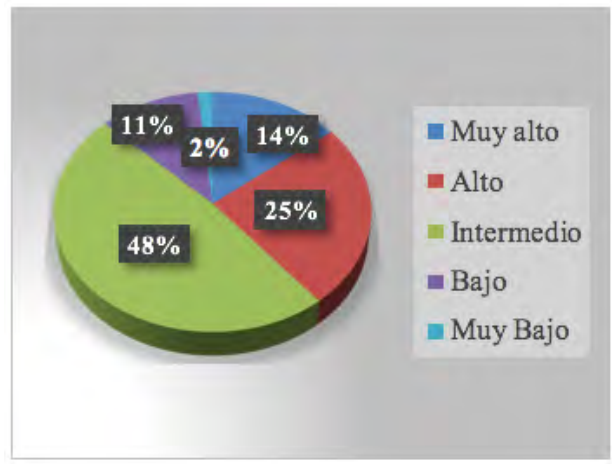

Figura 1. Nivel de apropiación de las TIC en docentes

Fuente: elaboración propia

De acuerdo con la tabla y resultados obtenidos a través de la encuesta de la pregunta 2, sobre qué nivel de apropiación de las TIC los docentes consideran que poseen, se puede visualizar que el $48 \%$ de los docentes considera tener un nivel intermedio de apropiación de las TIC y el $25 \%$ consideran tener un nivel Alto. Dados estos resultados se puede decir que el $73 \%$ de los docentes de la UG cuentan con un nivel intermedio-alto sobre el uso de las TIC, lo que indica que la mayoría tiene conocimiento medio sobre cómo manejar las diferentes aplicaciones de una manera correcta.

Una pregunta muy importante dentro de la investigación es acerca de las capacitaciones de las competencias digitales (Tabla 7), la cuales proveen de habilidades para hacer uso de las TIC. De acuerdo con el análisis de esta pregunta se pudo notar que el $67 \%$ de docentes ha recibido capacitaciones acerca de las Competencias Digitales por parte de la Universidad de Guayaquil antes del cambio de modalidad (Figura 2), lo cual indicaría que la mayoría de los docentes deberían tener conocimiento sobre el correcto uso de las TIC al momento de impartir las clases virtuales. 
Tabla 7.

Capacitaciones de competencias digitales

\begin{tabular}{|l|l|l|}
\hline Opción & Docentes encuestados & Porcentaje \\
\hline Sí & 165 & $67,1 \%$ \\
\hline No & 81 & $32,93 \%$ \\
\hline Total & 246 & $100 \%$ \\
\hline
\end{tabular}

Fuente: elaboración propia

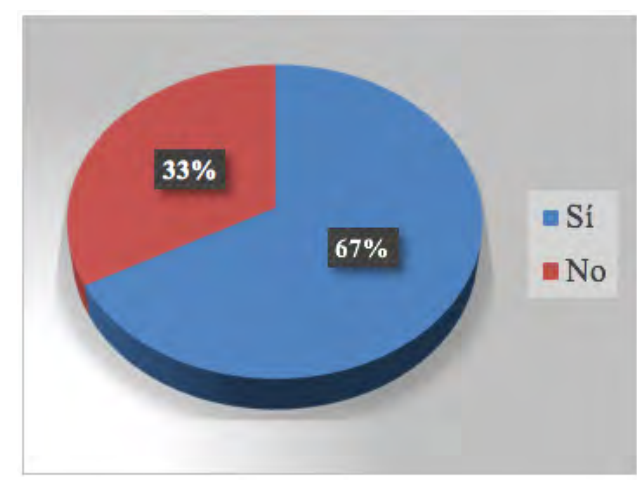

Figura 2. Capacitaciones de competencias digitales

Fuente: elaboración propia

De acuerdo con el análisis de la Tabla 8 y Figura 3 de la pregunta 9, en cuanto a lo que consideran que existe sobre interacción entre docente-estudiante en las clases virtuales vs clases presenciales, se obtuvo como resultados que el 33,74\% de los docentes consideran que el nivel de interacción se encuentra en un punto intermedio, y el 23,98\% consideran que el nivel de interacción con los estudiantes es bajo. Lo cual indica que no existe una buena interacción durante las clases virtuales entre los involucrados, lo que afecta directamente el proceso de enseñanzaaprendizaje.

Se utilizó una escala del 1 al 5, siendo el 5 el más alto.

Tabla 8.

Nivel de interacción entre docente-estudiante

\begin{tabular}{|l|l|l|}
\hline Opción & Docentes encuestados & Porcentaje \\
\hline 1 & 52 & $21,14 \%$ \\
\hline 2 & 59 & $23,98 \%$ \\
\hline 3 & 83 & $33,74 \%$ \\
\hline 4 & 34 & $13,82 \%$ \\
\hline 5 & 18 & $7,32 \%$ \\
\hline Total & 246 & $100 \%$ \\
\hline
\end{tabular}

Fuente: elaboración propia 


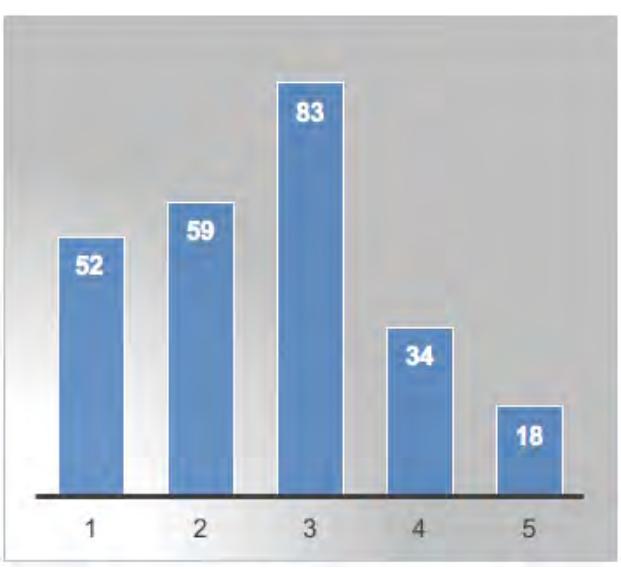

Figura 3. Nivel de interacción entre docente-estudiante

Fuente: elaboración propia

\subsection{Análisis e interpretación de resultados de la encuesta de estudiantes}

En cuanto a los estudiantes, se destaca la pregunta 5, debido a que no solo hace referencia a las clases virtuales, sino a la calidad de la educación. Lo cual dio como resultado que el $48 \%$ de estudiantes opinan que la calidad de educación en la UG es regular y otra de las respuestas con mayor porcentaje es $37 \%$ que opinan que la calidad de la educación en la UG es buena. Con los resultados obtenidos podemos notar que casi la mitad de los estudiantes opinan que la calidad de educación de la Universidad de Guayaquil es considerada por los estudiantes como regular, más no excelente (ver Tabla 9 y Figura 4).

Tabla 9.

Calidad de educación en la UG

\begin{tabular}{|l|l|l|}
\hline Opción & Estudiantes encuestados & Porcentaje \\
\hline Muy buena & 15 & $5,4 \%$ \\
\hline Buena & 103 & $37,7 \%$ \\
\hline Regular & 133 & $48,2 \%$ \\
\hline Mala & 17 & $6,2 \%$ \\
\hline Muy mala & 8 & $2,9 \%$ \\
\hline Total & 276 & $100 \%$ \\
\hline
\end{tabular}

Fuente: elaboración propia 


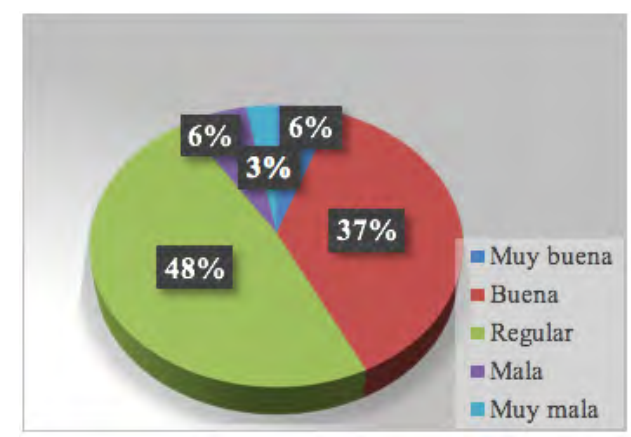

Figura 4. Calidad de educación en la UG

Fuente: elaboración propia

¿Ha recibido capacitaciones sobre competencias digitales por parte de la Universidad de Guayaquil? Fue otra de las preguntas que de acuerdo con el análisis se pudo notar que el 87,3\% de estudiantes nunca han recibido capacitaciones acerca de las competencias digitales por parte de la Universidad de Guayaquil (Tabla 10 y Figura 5), lo cual indicaría que la mayoría de los estudiantes no cuentan con el conocimiento sobre las competencias digitales y sobre cómo hacer un correcto uso de las TIC en las clases virtuales. Cabe destacar que de acuerdo a los resultados obtenidos en la pregunta 5 de docentes se nota una gran diferencia en los resultados, dado que un $67.1 \%$ recibieron capacitaciones y los estudiantes solo un $13 \%$.

Tabla 10.

Capacitaciones competencia digitales enestudiantes

\begin{tabular}{|l|l|l|}
\hline Opción & $\begin{array}{l}\text { Estudiantes } \\
\text { encuestados }\end{array}$ & Porcentaje \\
\hline Sí & 35 & $12,7 \%$ \\
\hline No & 241 & $87,3 \%$ \\
\hline Total & 276 & $100 \%$ \\
\hline
\end{tabular}

Fuente: elaboración propia

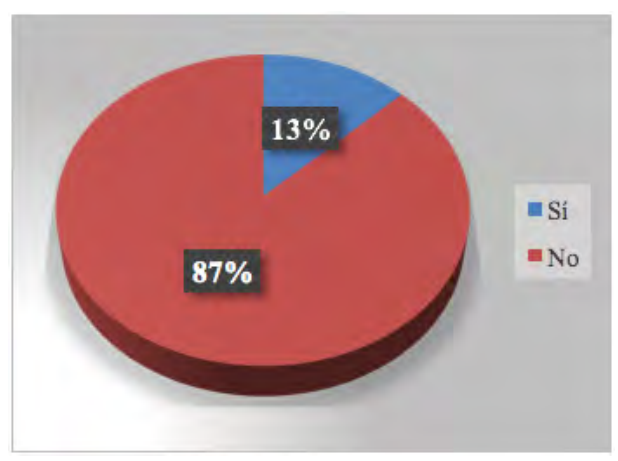

Figura 5. Capacitaciones competencia digitales enestudiantes

Fuente: elaboración propia 
Cuando se les consultó si consideraban que el nivel de aprendizaje está al mismo nivel que la educación presencial se obtuvieron como resultados que el $38,74 \%$ de los estudiantes consideran que el nivel de aprendizaje se encuentra en un punto intermedio, y el 26,98\% opinan que están en desacuerdo con respecto a que el nivel de aprendizaje de las clases virtuales está al mismo nivel que las clases presenciales (ver Tabla 11 y Figura 6). Lo cual indica que los estudiantes consideran que el nivel de aprendizaje se encuentra en un punto intermedio-bajo con respecto a las clases presenciales.

Tabla 11.

Nivel de aprendizaje modalidad en línea vs presencial

\begin{tabular}{|l|l|l|}
\hline Opción & Estudiantes encuestados & Porcentaje \\
\hline Totalmente de acuerdo & 17 & $6,2 \%$ \\
\hline De acuerdo & 50 & $18,1 \%$ \\
\hline Neutral & 105 & $38,0 \%$ \\
\hline En desacuerdo & 71 & $25,7 \%$ \\
\hline Totalmente en desacuerdo & 33 & $12,0 \%$ \\
\hline Total & 276 & $100,0 \%$ \\
\hline
\end{tabular}

Fuente: elaboración propia

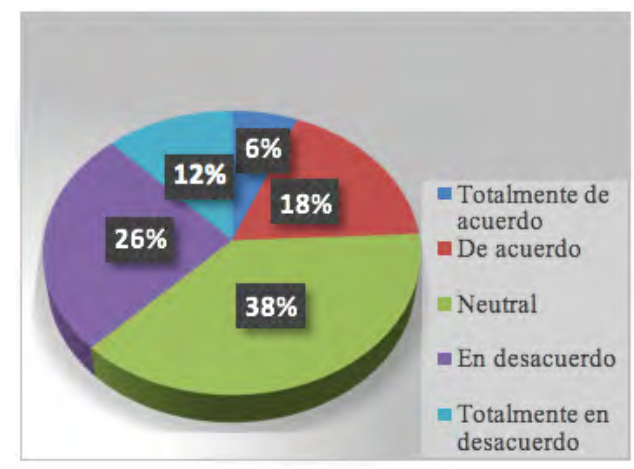

Figura 6. Nivel de aprendizaje modalidad en línea vspresencial

Fuente: elaboración propia

El análisis de la pregunta, sobre qué tipo de modalidad de estudio prefieren, dio como resultado que $78 \%$ de los estudiantes de la Universidad de Guayaquil prefieren la educación presencial, a pesar de contar con los recursos tecnológicos para acceder a las clases virtuales, tal como se evidencia en la Tabla 12 y la Figura 7. 
Tabla 12.

Modalidad de preferencia de los estudiantes

\begin{tabular}{|l|l|l|}
\hline Opción & $\begin{array}{l}\text { Estudiantes } \\
\text { encuestados }\end{array}$ & Porcentaje \\
\hline Modalidad en línea & 216 & $78,3 \%$ \\
\hline Modalidad presencial & 60 & $21,07 \%$ \\
\hline Total & 276 & $100 \%$ \\
\hline
\end{tabular}

Fuente: elaboración propia

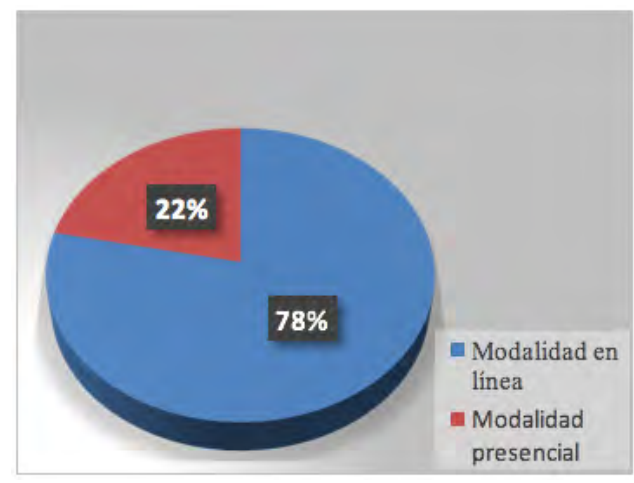

Figura 7. Modalidad de preferencia de los estudiantes

Fuente: elaboración propia

En términos generales, se puede decir que los docentes de las diferentes facultades de la Universidad de Guayaquil, según su criterio, cuentan con un nivel intermedio-alto de apropiación de las TIC, a pesar de que cierto porcentaje de docentes no fueron capacitados sobre las competencias digitales antes de la pandemia de la COVID-19, además, los docentes consideran que existe un nivel intermedio a bajo de interacción entre docentes-estudiantes, lo que demuestra cierta deficiencia en la capacitación docente para hacer uso de las tecnologías de la información dentro del desarrollo de sus actividades académicas. Habría que analizar las causas de la insatisfacción en las capacitaciones en TIC expresadas por los docentes para implementar los correctivos apropiados y mejorar los planes de capacitación en el futuro.

Por otra parte, los estudiantes de las diferentes facultades de la universidad caso de estudio consideran que la calidad de la educación de la institución es regular a buena, también manifestaron en su mayoría que no han recibido capacitaciones sobre las competencias digitales por parte de la universidad, además, los estudiantes estiman que el nivel de aprendizaje en la modalidad en línea se encuentra en un rango entre intermedio a bajo con respecto a la educación presencial, y por último, la mayoría de estudiantes prefieren la modalidad presencial.

No considerar apropiadas las capacitaciones en el uso de TIC pudiera traer como consecuencia un aprovechamiento parcial de las tecnologías en el proceso formativo, debido a que no tienen verdaderas competencias digitales. Puede considerarse que esto también está asociado con la calidad y cantidad de las capacitaciones que ofrece la Institución a sus docentes. 


\section{Conclusiones}

Mediante la revisión bibliográfica de elementos relacionados con las TIC, se logró determinar que estas herramientas son viables para aplicarlas en el ámbito educativo, particularmente dirigidas a la educación superior; para esto se debe adquirir competencias digitales o tener un nivel de apropiación de las TIC intermedio para hacer un correcto uso y apropiación de las mismas.

De acuerdo a las encuestas realizadas a los docentes y estudiantes de la Universidad de Guayaquil se evidenció que tanto la institución, como las personas que la conforman, no se encontraban preparadas para el cambio repentino de modalidad de clases, pese a que los docentes y estudiantes tienen conocimientos de las TIC; y poseen los recursos tecnológicos, eso no fue suficiente para lograr que el proceso de enseñanza-aprendizaje sea óptimo debido a que no existió una buena planificación, existen ciertas dificultades en el uso de las plataformas, lo que ocasiona que no se dé una buena interacción durante las clases sincrónicas entre los docentes y estudiantes.

Tomando en cuenta los puntos más relevantes de la investigación, se puede determinar que las TIC tienen un gran impacto en la modalidad en línea debido a que se visualizó un incremento sustancial en su implementación en las clases virtuales frente al tiempo pre-cuarentena, donde su uso era limitado durante el proceso de enseñanza- aprendizaje. Se deben hacer uso de las diferentes herramientas tecnológicas fuera de las propiciadas por la Universidad de Guayaquil, para lograr una mayor interacción entre estudiantes y docentes al momento de realizar las clases en línea.

Se puede concluir que, en general, la percepción que tienen los docentes y estudiantes en cuanto al impacto del uso de TIC en los procesos de enseñanza aprendizaje es muy positiva, lo que es un importante punto a favor para que la Universidad de Guayaquil aproveche esa apertura de sus docentes y estudiantes y les proporcione las herramientas tecnológicas apropiadas se puedan implementar en el desarrollo del proceso enseñanza aprendizaje. No obstante, se requiere que la universidad revise los mecanismos de capacitación en TIC y más aún en este momento en el cual dada la situación de la pandemia por la COVID-19, se ha tenido que migrar completamente al uso de TIC para desempeñar las actividades académicas de toda la Universidad. 


\section{Referencias}

Asamblea Nacional Constituyente de Ecuador. (2008). Constitución de la República del Ecuador.

Contreras, J. L. R., Pabón, J. C. R., \& Ríos, G. M. V. (2017). Importancia de las Tic en enseñanza de las matemáticas. Revista MATUA ISSN: 2389-7422, 4(2).

García, M., Reyes, J., \& Godínez, G. (2018). Las TIC en la educación superior, innovaciones y retos / The ICT in higher education, innovations and challenges. RICSH Revista Iberoamericana de las Ciencias Sociales y Humanísticas, 6(12), párr.17.https://doi.org/10.23913/ricsh.v6i12.135

Hernández-Sampieri, R. y Mendoza-Torres, C. (2018). Metodología de la Investigación. Editorial Mc Graw-Hill: México.

Levano, L., Sanchez, S., Guillén, P., Tello, S., Herrera, N., \& Collantes, Z. (2019). Competencias digitales y educación. La Scientific Electronic Library Online, 7(2). http://dx.doi.org/10.20511/pyr2019.v7n2.329

Ley Orgánica de Educación Intercultural. (2011).

Ley Orgánica de Educación Intercultural. (2017).

López, D., González Orellana, M., García, E., Ramirez de Bello Suazo, A. L., Urrutia, J., Escobar Salmerón, J. E., \& Saz, C. (2020). COVID-19: Una mirada interdisciplinaria a la pandemia.

Mosquera, I. (2018). El necesario desarrollo de la competencia digital del profesorado. UNIR Revista. https:// www.unir.net/educacion/revista/el-necesario-desarrollo-de-la-competencia-digital-del-profesorado/

Reglamento general a la Ley Orgánica de Educación superior. 2019. Decreto Ejecutivo 742.

UNESCO. (2020). Coalición Mundial para la Educación COVID-19. https://es.unesco.org/covid19/globaleducationcoalition

Universidad de Guayaquil. (2020a, mayo). Memorando Nro. UG-CFAP-2020-0980-M. 
Copyright (c) 2021 Janeth Pilar Díaz Vera, Alicia Karina Ruiz Ramírez y Carolina Egüez Cevallos

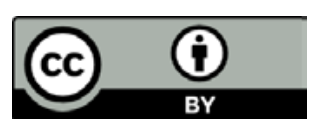

Este texto está protegido bajo una licencia internacional Creative Commons 4.0.

Usted es libre para Compartir-copiar y redistribuir el material en cualquier medio o formato

- y Adaptar el documento - remezclar, transformar y crear a partir del material-para cualquier propósito, incluso para fines comerciales, siempre que cumpla las condiciones de Atribución. Usted debe dar crédito a la obra original de manera adecuada, proporcionar un enlace a la licencia, e indicar si se han realizado cambios. Puede hacerlo en cualquier forma razonable, pero no de forma tal que sugiera que tiene el apoyo del licenciante o lo recibe por el uso que hace de la obra.

$\underline{\text { Resumen de licencia - Texto completo de la licencia }}$ 